\title{
Refractive index measurements of solid parahydrogen
}

\author{
Manori Perera, ${ }^{1,9}$ Brian A. Tom, ${ }^{1,2}$ Yuki Miyamoto,${ }^{3,4}$ Michael W. Porambo, ${ }^{1}$ Lauren E. Moore,${ }^{1,5}$ \\ William R. Evans, ${ }^{6}$ Takamasa Momose, ${ }^{3,7}$ and Benjamin J. McCall ${ }^{1,6,8,10}$ \\ ${ }^{1}$ Department of Chemistry, University of Illinois at Urbana-Champaign, 600 South Mathews Avenue, Urbana, Illinois 61801, USA \\ ${ }^{2}$ Currently with the Department of Chemistry, United States Air Force Academy, Colorado 80840, USA \\ ${ }^{3}$ Department of Chemistry, Kyoto University, Kyoto 606-8502, Japan \\ ${ }^{4}$ Currently with Graduate School of Natural Science and Technology, Okayama University, 3-1-1, Tsushima-Naka, \\ Okayama 700-8530, Japan \\ ${ }^{5}$ Currently with Celanese Corporation, 9502 Bayport Boulevard, Pasadena, Texas 77507, USA \\ ${ }^{6}$ Department of Physics, University of Illinois at Urbana-Champaign, 1110 West Green Street, Urbana, Illinois 61801, USA \\ ${ }^{7}$ Currently with Department of Chemistry, University of British Columbia, 2036 Main Mall, Vancouver, \\ British Columbia V6T 1Z1, Canada \\ ${ }^{8}$ Department of Astronomy, University of Illinois at Urbana-Champaign, 1002 West Green Street, Urbana, Illinois 61801, USA \\ ${ }^{9}$ e-mail: mperera@illinois.edu \\ ${ }^{10}$ e-mail: bjmccall@illinois.edu
}

Received December 22, 2010; accepted January 31, 2011;

posted February 11, 2011 (Doc. ID 140160); published March 9, 2011

Solid para- $\mathrm{H}_{2}$ is a promising gain medium for stimulated Raman scattering, due to its high number density and narrow Raman linewidth. In preparation for the design of a cw solid hydrogen Raman laser, we have made the first measurements, to our knowledge, of the index of refraction of a solid para- $\mathrm{H}_{2}$ crystal, in the wavelength range of $430-1100 \mathrm{~nm}$. For a crystal stabilized at $4.4 \mathrm{~K}$, this refractive index is measured to be $n_{p-\mathrm{H}_{2}}=1.130 \pm 0.001$ at $514 \mathrm{~nm}$. A slight, but significant, dependence on the final crystal-growth temperature is observed, with higher $n_{p-\mathrm{H}_{2}}$ at higher crystal-growth temperatures. Once a crystal is grown, it can be heated up to $10 \mathrm{~K}$ with no change in $n_{p-\mathrm{H}_{2}}$. The refractive index varies only slightly over the observed wavelength range, and no significant birefringence was observed. (C) 2011 Optical Society of America

OCIS codes: $290.3030,120.4530,260.1180,160.4760,290.5910$.

Molecular hydrogen has long been considered a particularly useful gain medium for stimulated Raman scattering, because of its large vibrational frequency. As early as 1986, sequential Stokes conversion in a multipass cell of gaseous $\mathrm{H}_{2}$ was used to convert a pulsed dye laser into a continuously tunable laser in the $1-12 \mu \mathrm{m}$ region [1]. Similar setups have been used successfully by many groups, but they depend on multipass mirrors with both high damage thresholds and high reflectivity over a wide wavelength range, which are difficult to obtain.

Solid para- $\mathrm{H}_{2}$ has recently been demonstrated to be a suitable medium for Raman shifting pulsed lasers, without the need for specialized optics. Because of its higher density $\left(n \sim 2.6 \times 10^{22} \mathrm{~cm}^{-3}\right)$ and smaller Raman linewidth ( $\Gamma \sim 7 \mathrm{MHz}$ [2]), solid $\mathrm{H}_{2}$ would be expected to have a much higher Raman gain than gaseous $\mathrm{H}_{2}$. Indeed, measurements by Katsuragawa and Hakuta [3] have shown that the solid's Raman gain coefficient is $70 \overline{0} 0$ times higher than that of the gas. In the last decade, solid $\mathrm{H}_{2}$ Raman pulsed lasers have been demonstrated [3-5] and are now in routine use in spectroscopic applications [6,7].

However, the high resolution spectroscopic study of molecular vibrations requires $\mathrm{cw}$ lasers with narrower bandwidth than can be obtained by transform-limited pulsed lasers. Carlsten et al. [8] have demonstrated a cw Raman laser in gaseous $\mathrm{H}_{2}$, using a high-finesse cavity to produce sufficient intracavity power to drive the stimulated Raman scattering. Their lasers have achieved wide tunability [9] and high conversion efficiency [10], but are technically complicated due to the need for a high-finesse cavity. To our knowledge, these lasers have not been used by other groups.
Our long-term goal is to develop a cw Raman laser based on solid para- $\mathrm{H}_{2}$. The high Raman gain of the solid should permit such a laser to have a considerably lower finesse cavity than the gaseous $\mathrm{H}_{2}$ lasers, thereby making them easier to align and maintain. The high gain of the solid may also facilitate the extension of the cw Raman $\mathrm{H}_{2}$ laser to longer wavelengths, where the stimulated Raman scattering process is less efficient.

To design such a laser, it is essential to minimize Fresnel losses at interfaces inside the laser cavity, in particular between the solid $\mathrm{H}_{2}$ gain medium and the cell windows (typically sapphire) that contain the $\mathrm{H}_{2}$ crystal. The angles of these interfaces can be chosen to be at or near Brewster's angle for minimal reflective losses, but this requires knowledge of the refractive index of solid para- $\mathrm{H}_{2}$; to our knowledge, this quantity (which we refer to as $n_{p-\mathrm{H}_{2}}$ ) has not been previously measured. In this Letter, we describe the procedure used independently in our labs in Illinois and Kyoto to generate a uniform para- $\mathrm{H}_{2}$ crystal and to measure $n_{p-\mathrm{H}_{2}}$. The dependence of $n_{p-\mathrm{H}_{2}}$ on wavelength and the polarization angle of light, as well as the temperature of the crystal, is also discussed.

Solid para- $\mathrm{H}_{2}$ crystals were grown in a cell (illustrated in Fig. 1) consisting of a $1.27 \mathrm{~cm}$ hole bored in a $5.1 \mathrm{~cm}$ stainless steel body. One end of the cell was machined at an angle $\left(\phi=10.0^{\circ}\right.$ in Illinois; $\phi=20.2^{\circ}$ in Kyoto) to induce a deflection in a laser beam propagating through the crystal; the measurement of this deflection enabled the measurement of $n_{p-\mathrm{H}_{2}}$. The ends of the cell were sealed with sapphire windows using indium gaskets. The cell was mounted to an oxygen-free high-conductivity (OFHC) copper plate machined with a small pin that 


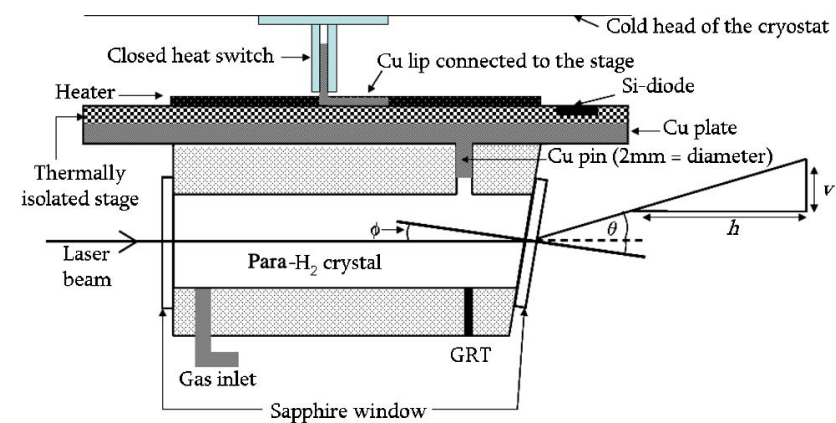

Fig. 1. (Color online) Schematic of the cell and the laser path in the presence of the para- $\mathrm{H}_{2}$ crystal.

protrudes into the cell body (sealed with an indium gasket), to serve as a nucleation point for crystal growth. The OFHC plate in turn was mounted to the thermally isolated stage of a liquid-helium-cooled cryostat.

The temperature of the cell was controlled by adjusting the thermal contact with the cold head by opening a heat switch, and/or by using a resistive heater on the cell. The temperature gradient within the cell was monitored using a germanium resistance thermometer (GRT) mounted on the bottom of the cell and a silicon diode mounted on the thermally isolated stage where the cell rests. The temperature difference between the two sensors was typically less than $1 \mathrm{~K}$, and because the thermal conductivity of solid para- $\mathrm{H}_{2}\left(1.5 \mathrm{~W} \mathrm{~cm}^{-1} \mathrm{~K}^{-1}\right.$ at $4 \mathrm{~K}$ [11] $)$ is so much higher than that of stainless steel $\left(0.003 \mathrm{~W} \mathrm{~cm}^{-1} \mathrm{~K}^{-1}[12]\right)$, the $\mathrm{H}_{2}$ crystal would be expected to be at a temperature very close to that of the silicon diode. The cell was filled through narrow stainless steel tubing, which passed along the radiation shield of the cryostat before connecting to the cell body.

The crystal was grown with $99.98 \%$ pure para- $\mathrm{H}_{2}[13]$ following the pressurized crystal-growth method [14], which is known to yield a transparent single crystal. Para- $\mathrm{H}_{2}$ gas was condensed into the cell at $15-20 \mathrm{~K}$, while the liquid level was watched. Once the cell was filled, the cryostat's gas line was connected to a small $(150 \mathrm{~mL})$ tank and the cell was heated to $30-45 \mathrm{~K}$ to boil off the liquid and pressurize the tank. Fresh para- $\mathrm{H}_{2}$ gas was then condensed into the cell, and, finally, pressurized gas from the small tank ( $\sim 200$ psi in Illinois, $\sim 290$ psi in Kyoto) was applied to the condensed para- $\mathrm{H}_{2}$ liquid. The temperature was slowly lowered to the final temperature over a $1 \mathrm{~h}$ period as the crystal grew. The crystallization was initiated from the coldest point (the copper pin at the top of the cell) and the crystal growth was observed as the liquid-solid boundary (crystallizing surface) slowly moved downward. The same procedure was repeated to create crystals with higher final growth temperatures, which required lower backing pressures [15].

Hydrogen's ability to yield such a large single crystal stems from the fact that solid para- $\mathrm{H}_{2}$ is a quantum crystal, with a "self-annealing" property, similar to solid helium [16]. We observed a similar liquid-solid boundary during crystal growth as in previous studies ([14], Fig. 2), and our observation of a single crystallizing surface is strong evidence that a single crystal was in fact formed. In other trial runs (with different crystal-growth procedures, inadequate backing pressure, or impurities in the sample), a single crystallizing surface was not ob-

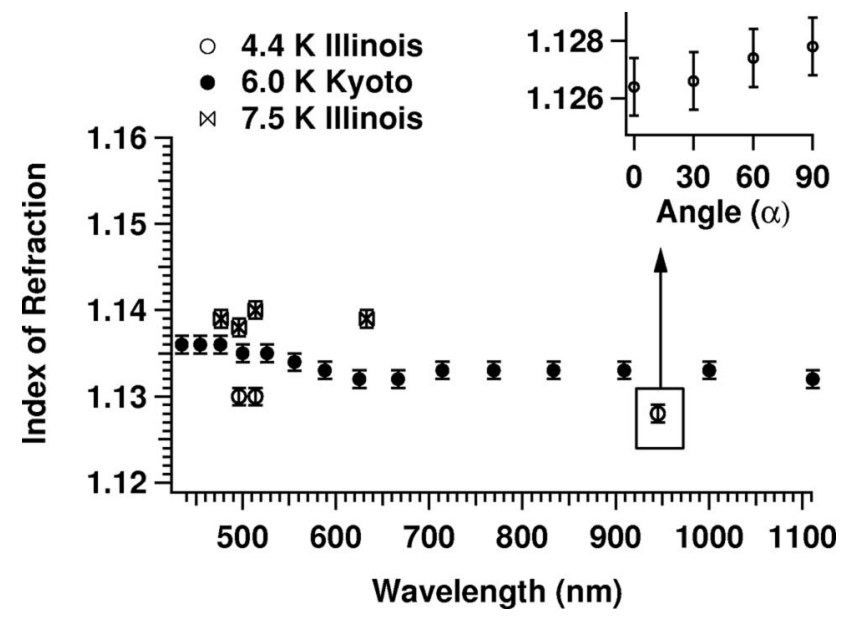

Fig. 2. Index of refraction data taken at various growth temperatures of the crystal. The inset shows the dependence on $\alpha$, the angle (in degrees) between the polarization axis of the light and the $c$ axis of the crystal.

served: the resulting samples were not completely transparent; laser beams sent through those samples suffered complex scattering patterns, and cracks would result as the temperature dropped, separating individual crystals. With proper care in the crystal-growth process, however, a transparent single crystal always resulted. Previous work has established that high purity para- $\mathrm{H}_{2}$ crystallizes to form a hexagonal close-packed lattice at low temperature and high pressure [15] and that the $c$ axis follows the largest thermal gradient $[\underline{4}, \underline{17}]$; in the present case this is the vertical axis.

The optical setup used for measurements of $n_{p-\mathrm{H}_{2}}$ in Illinois consisted of three lasers: an argon ion laser with 476, 496, and $514 \mathrm{~nm}$ emission; a $\mathrm{He}-\mathrm{Ne}$ laser with $632 \mathrm{~nm}$ emission; and a tunable diode laser at $945 \mathrm{~nm}$. After the crystal was fully grown, the laser light was passed through the crystal. The angle of the deflected beam $(\theta)$ was determined by measuring its distance (labeled $v$ ) from the horizontal at two positions separated by $1.5 \mathrm{~m}$ (labeled $h$ ), as shown in Fig. 1 . The beam position was determined by monitoring the voltage of a photodiode as it was translated vertically. The measured beam positions are accurate to within $\pm 0.013 \mathrm{~cm}$, the precision of the micrometer mount used. In Kyoto, a master oscillator power oscillator laser was used to measure $n_{p-\mathrm{H}_{2}}$ at 15 different wavelengths between $430 \mathrm{~nm}$ and $1100 \mathrm{~nm}$. In that measurement, the deflected angle $(\theta)$ was measured by locating the laser spot (visually) on a screen $6.5 \mathrm{~m}$ from the cryostat.

Snell's law, $n_{p-\mathrm{H}_{2}} \sin (\phi)=n_{\mathrm{vac}} \sin (\theta+\phi)$, was used to infer $n_{p-\mathrm{H}_{2}}$. Here, $\phi$ includes both the tilt of the cryostat stage with respect to the incident laser beam as well as the angle of the intentionally tilted window. The dependence of $n_{p-\mathrm{H}_{2}}$ on the polarization angle of the incident laser was measured by inserting a half-wave plate in the laser path prior to the cryostat and changing its angle in increments of $15^{\circ}$. The results are presented in Fig. 2 .

The measurements show only a slight dependence of $n_{p-\mathrm{H}_{2}}$ on wavelength. The $6.0 \mathrm{~K}$ data, which have the largest wavelength coverage, show that $n_{\mathrm{p}-\mathrm{H}_{2}}$ decreases slightly with increasing wavelength up to $\sim 650 \mathrm{~nm}$ and then levels off, becoming consistent with a constant 
value of $1.132 \pm 0.001$. The $4.4 \mathrm{~K}$ and $7.5 \mathrm{~K}$ data sets are each consistent with a constant $n_{p-\mathrm{H}_{2}}$, but these data have sparser wavelength coverage. The constancy of $n_{p-\mathrm{H}_{2}}$ with wavelength is expected, since para- $\mathrm{H}_{2}$ has no absorptions between the far-IR and the UV.

The inset of Fig. 2 shows how $n_{p-\mathrm{H}_{2}}$ varies with the polarization angle, $\alpha$, of the incoming laser beam (at $945 \mathrm{~nm}$ and $4.4 \mathrm{~K}$ ). Although there is a hint of an increase of $n_{p-\mathrm{H}_{2}}$ with $\alpha$, the trend is not statistically significant, given our measurement uncertainties; we adopt an upper limit of 0.002 for the magnitude of birefrigence.

The data indicate that $n_{p-\mathrm{H}_{2}}$ increases with the final temperature of crystal growth. This is surprising, as the density of solid para- $\mathrm{H}_{2}$ is known $[14,15]$ to decrease slightly with increasing temperature in this range. One might therefore anticipate that $n_{p-\mathrm{H}_{2}}$ would decrease with decreasing density (as the temperature increases), but our observations show that $n_{p-\mathrm{H}_{2}}$ instead increases.

To test the sensitivity of $n_{p-\mathrm{H}_{2}}$ to the rate of crystal growth, we grew a crystal at a faster rate, lowering the temperature to $4.4 \mathrm{~K}$ in only $10 \mathrm{~min}$, rather than $1 \mathrm{~h}$. This crystal had a slightly higher $n_{p-\mathrm{H}_{2}}$ (at $514 \mathrm{~nm}$ ) of $1.134 \pm 0.001$, compared with $1.130 \pm 0.001$ for the slowly grown crystal. One might expect the slowly grown crystal to attain a higher density than the quickly grown crystal, as the former has more time to rearrange its lattice. These observations are consistent with the other measurements, in the sense that in both cases $n_{p-\mathrm{H}_{2}}$ increases with decreasing density (due to either higher growth temperature or faster growth rate).

After crystal growth is complete, raising the temperature does not change $n_{p-\mathrm{H}_{2}}$. For a crystal stabilized at a final growth temperature of $4.4 \mathrm{~K}$, we gradually increased the temperature up to $10 \mathrm{~K}$ (in increments of $\sim 0.5 \mathrm{~K}$ ) and observed no change in the deflection of the laser. Once the crystal is completely grown, the lattice cannot expand because it is completely confined in the cell, and the constant density leads to a constant $n_{p-\mathrm{H}_{2}}$.

The observed temperature dependence of $n_{p-\mathrm{H}_{2}}$ is significantly larger than can be accounted for by the measurement uncertainties. For example, in order for the measured $n_{p-\mathrm{H}_{2}}$ of the $4.4 \mathrm{~K}$ crystal to fall within the uncertainties of the data at $6.0 \mathrm{~K}$, the measured deflection angle would have to have been mismeasured by $0.14^{\circ}$, which corresponds to an error in the vertical beam deflection $(v)$ of $0.23 \mathrm{~cm}$, or 18 times our precision. We therefore conclude that the surprising inverse dependence of $n_{p-\mathrm{H}_{2}}$ on density is not due to systematic errors in the measurements, but we cannot offer a physical explanation of the effect at this time.

In summary, we have measured the refractive index of solid para- $\mathrm{H}_{2}$ for the first time, to our knowledge.
Measurements were performed for final crystal-growth temperatures of $4.4,6.0$, and $7.5 \mathrm{~K}$, over a wavelength range of $430-1100 \mathrm{~nm}$. The refractive index (at $514 \mathrm{~nm}$ ) is $n_{p-\mathrm{H}_{2}}=1.130 \pm 0.001$ for a crystal stabilized at $4.4 \mathrm{~K}$ and increases to $1.140 \pm 0.001$ at $7.5 \mathrm{~K}$. Our measurements suggest that $n_{p-\mathrm{H}_{2}}$ is dependent on the density of the crystal, with higher values at lower densities. A small dependence of $n_{p-\mathrm{H}_{2}}$ on wavelength was observed, but no significant dependence on polarization was seen. These measurements can be expected to facilitate the development of a cw Raman laser using solid para- $\mathrm{H}_{2}$ as the gain medium, which may ultimately lead to a new widely tunable and narrow linewidth laser source in the midIR region.

The authors wish to thank Siddhartha Bhasker for his assistance with the early stages of the experimental development in Illinois. The work in Illinois has been supported by the David and Lucile Packard Foundation and by the University of Illinois.

\section{References}

1. P. Rabinowitz, B. N. Perry, and N. Levinos, IEEE J. Quantum Electron. 22, 797 (1986).

2. T. Momose, D. P. Weliky, and T. Oka, J. Mol. Spectrosc. 153, 760 (1992).

3. M. Katsuragawa and K. Hakuta, Opt. Lett. 25, 177 (2000).

4. M. Fushitani, S. Kuma, Y. Miyamoto, H. Katsuki, T. Wakabayashi, T. Momose, and A. F. Vilesov, Opt. Lett. 28, 37 (2003).

5. B. J. McCall, A. J. Huneycutt, R. J. Saykally, C. M. Lindsay, T. Oka, M. Fushitani, Y. Miyamoto, and T. Momose, Appl. Phys. Lett. 82, 1350 (2003).

6. K. E. Kuyanov, T. Momose, and A. F. Vilesov, Appl. Opt. 43, 6023 (2004).

7. H. Hoshina, D. Skvortsov, B. G. Sartakov, and A. F. Vilesov, J. Chem. Phys. 132, 074302 (2010).

8. J. K. Brasseur, K. S. Repasky, and J. L. Carlsten, Opt. Lett. 23, 367 (1998)

9. L. S. Meng, K. S. Repasky, P. A. Roos, and J. L. Carlsten, Opt. Lett. 25, 472 (2000).

10. L. S. Meng, P. A. Roos, K. S. Repasky, and J. L. Carlsten, Opt. Lett. 26, 426 (2001).

11. R. G. Bohn and C. F. Mate, Phys. Rev. B 2, 2121 (1970).

12. NIST Cryogenic Technologies Group, Material Properties Compilation, available at: http://cryogenics.nist.gov.

13. B. A. Tom, S. Bhasker, Y. Miyamoto, T. Momose, and B. J. McCall, Rev. Sci. Instrum. 80, 016108 (2009).

14. M. Suzuki, M. Katsuragawa, R. S. D. Sihombing, J. Z. Li, and K. Hakuta, J. Low Temp. Phys. 111, 463 (1998).

15. P. C. Souers, Hydrogen Properties for Fusion Energy (University of California, 1986).

16. T. Momose, J. Low Temp. Phys. 111, 469 (1998).

17. D. P. Weliky, K. E. Kerr, T. J. Byers, Y. Zhang, T. Momose, and T. Oka, J. Chem. Phys. 105, 4461 (1996). 